\title{
Association of solar wind proton flux extremes with pseudostreamers
}

\author{
L. Zhao, ${ }^{1,2}$ S. E. Gibson, ${ }^{1}$ and L. A. Fisk ${ }^{2}$ \\ Received 20 January 2013; revised 8 May 2013; accepted 9 May 2013; published 17 June 2013.
}

[1] We investigate the characteristics and solar origins of a subpopulation of the solar wind that possesses extreme values of proton flux. Ulysses observations including solar wind magnetic flux, proton flux, number density and velocity, and ionic composition are examined in this study. We find that the departures of solar wind proton flux from its constancy occur for time intervals leading up to and encompassing the past two solar minima, and the extreme-proton-flux wind possesses the following characteristics: (1) it generally originates from sources middle-distant from the Heliospheric Current Sheet (HCS); (2) it is associated with a broad range of velocities and electron temperatures but excludes very fast/cold wind; (3) it exhibits anticorrelation between electron temperature and proton velocity, as does the rest of the solar wind; (4) it has extreme proton density values relative to the rest of the solar wind; and (5) the extreme-high-proton-flux wind has radial component of open magnetic flux $(\mathrm{Br})$ greater than the rest of the solar wind, and both extreme-high and extreme-low wind do not possess the lowest values of $\mathrm{Br}$ flux. Comparing with SOHO EIT $195 \AA$ coronal images, we find the observed extreme-proton-flux wind has temporal and spatial coincidence with the appearance of low-latitude coronal holes present in the recent two solar minima; the magnetic field lines extrapolated by the Potential Field Source Surface model confirm there are coronal pseudostreamer structures involved. So we propose that these extreme-proton-flux winds can be associated with mid- to low-latitude coronal holes and "pseudostreamer" structures.

Citation: Zhao, L., S. E. Gibson, and L. A. Fisk (2013), Association of solar wind proton flux extremes with pseudostreamers, J. Geophys. Res. Space Physics, 118, 2834-2841, doi:10.1002/jgra.50335.

\section{Introduction}

[2] The "traditional" picture of solar minimum is that of a dipole magnetic configuration, with closed magnetic loops at the solar equator and open magnetic field at the poles. Figure 1 is a cartoon in which the simplicity of the magnetic dipole minimum is illustrated (Figure 1a). This picture seemed to hold true during the solar cycle 22-23 minimum, and the solar wind also appeared, at least to the first order, to have a bimodal structure. Figure 2 shows Ulysses' polar plots in its first and third orbits covering the recent two solar minima (Figures $2 \mathrm{a}$ and $2 \mathrm{c}$ cover the cycle 22-23 minimum, and Figures $2 \mathrm{~b}$ and $2 \mathrm{~d}$ cover the cycle 23-24 minimum). Solar wind proton speed is polar plotted along the Ulysses' heliographic latitude and color coded by the solar wind ionic oxygen charge-state ratio, $\mathrm{O}^{7+} / \mathrm{O}^{6+}$, in Figures $2 \mathrm{a}$ and $2 \mathrm{~b}$. Because the solar wind ionic charge composition is frozenin at several solar radii, it can reflect the electron temperature

\footnotetext{
${ }^{1} \mathrm{NCAR} / \mathrm{HAO}$, Boulder, Colorado, USA.

${ }^{2}$ Department of Atmospheric, Oceanic and Space Sciences, University of Michigan, Ann Arbor, Michigan, USA.

Corresponding author: L. Zhao, NCAR/HAO, PO Box 3000, Boulder CO 80307-3000,USA. (lzh@ucar.edu, 1zh@umich.edu)

(C)2013. American Geophysical Union. All Rights Reserved. 2169-9380/13/10.1002/jgra.50335
}

in the coronal base of the solar wind origin [Hundhausen, 1972; Landi et al., 2012]. (Note that this freeze-in temperature is not to be confused with the solar wind electron kinetic temperature which we do not discuss in this paper.) Therefore, the $\mathrm{O}^{7+} / \mathrm{O}^{6+}$ has been long used as an indicator of the electron temperature at the coronal source of the solar wind [Burgi and Geiss, 1986; Zurbuchen et al., 2000] and has been used to differentiate the solar wind types by their different origins [von Steiger et al., 2001; Zurbuchen et al., 2002; Zhao et al., 2009]. Figures 2c and 2d show polar plot of solar wind proton flux, also color coded by $\mathrm{O}^{7+} / \mathrm{O}^{6+}$ ratio. In Figure 2a, we can see fast and cold (in electron temperature, as indicated by $\mathrm{O}^{7+} / \mathrm{O}^{6+}$ ) solar wind was observed to emanate from open magnetic flux at the polar coronal holes [Smith and Balogh, 1995; Dwivedi et al., 2000; Smith and Balogh, 2008]. At the heliospheric current sheet (HCS) above the closed magnetic loops in low-latitude regions, the solar wind had slow speed and hot electronic temperature (higher value of $\mathrm{O}^{7+} / \mathrm{O}^{6+}$ ) compared to the polar-coronalhole-associated wind [McComas et al., 2000; von Steiger et al., 2000; Zhao et al., 2009]. The transition between the fast polar-hole-associated wind to the rest of the wind was very sharp and occurred at approximately $30^{\circ}$ heliographic latitude [Gosling, 1997].

[3] However, in the recent solar cycle 23-24 minimum, the coronal magnetic configuration was clearly more complex than the traditional dipole (Figure 1b). The size of the 

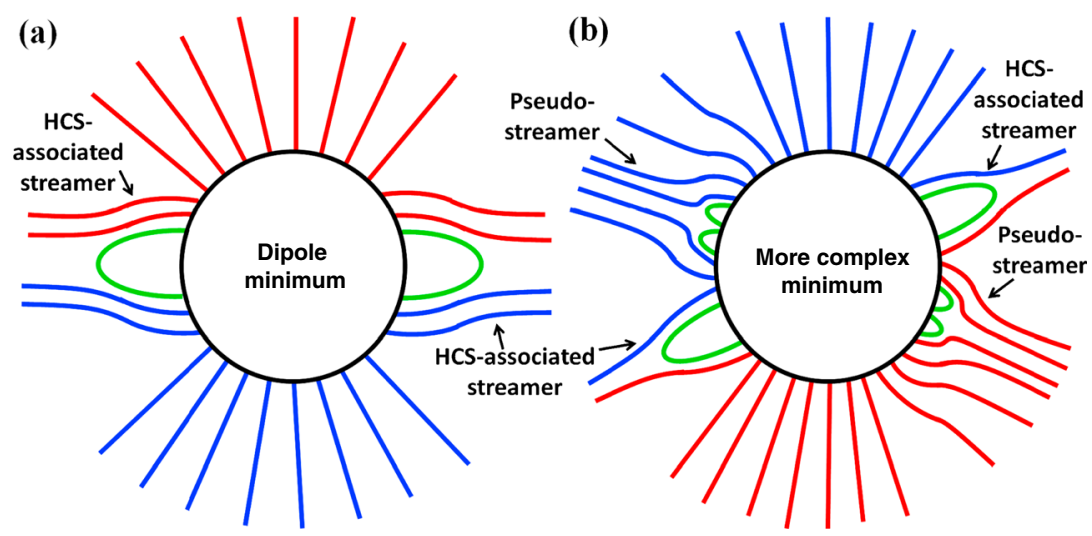

Figure 1. (a) Magnetic topologies characteristic of dipole solar minimum versus (b) more complex (and more realistic) configuration. Red (blue) lines indicate open magnetic field going outward from (toward) the Sun, and green lines represent closed magnetic loops.

polar coronal holes had shrunk [Zhao and Fisk, 2011; Kirk et al., 2009], and in addition to polar open field regions, middle- to low-latitude coronal holes were prevalent between 2007 and 2009 [Abramenko et al., 2010; de Toma, 2011]. As a result, unipolar streamer structures were also common during these years [Riley and Luhmann, 2012; Crooker et al., 2012; Wang et al., 2012]. A unipolar, or so-called "pseudo" streamer consists of two sets of closed magnetic loops that separate open flux with the same polarity [Hundhausen, 1972; Wang et al., 2007; Zhao and Webb, 2003], as opposed to the HCS-associated helmet streamer that separates the oppositely directed open magnetic flux regions (see Figure $1 \mathrm{~b}$ ).

[4] This more complex magnetic morphology resulted in a more complex solar wind structure (Figure 2b). The fastest and coldest wind was still associated with the polar coronal holes far from the HCS (more than $50^{\circ}$ heliographic latitude), and slow and hot wind still was associated with the HCS. However, solar wind with intermediate velocity was observed at low to middle latitudes, and the transition between these two populations was gradual and less bimodal [Gibson and Zhao, 2012, and references therein; Zhao et al., 2012].

[5] It is the purpose of this paper to examine the implications of different magnetic configurations in the corona for the solar wind properties in the recent two minima. In section 2, we use Ulysses observations to investigate solar wind dynamic properties including the proton flux, velocity and density, electron temperature (as indicated by $\mathrm{O}^{7+} / \mathrm{O}^{6+}$ ratio), and angular normal distance (Ds) from the solar wind source to the HCS at the coronal source surface, and we find that there is an interesting subset of the solar wind that possesses extreme-high/low-proton-flux values. In section 3, we examine two Carrington rotations (CRs) to illustrate the possible association between the extreme-proton-flux wind and the pseudostreamers in the solar corona. One $\mathrm{CR}$ is chosen from each solar minimum, when there are extremeproton-flux winds observed by Ulysses while it passes between low-latitude coronal hole and polar coronal holes as shown by SOHO EIT $195 \AA$ coronal images. Potential field source surface (PFSS) extrapolations indicate the presence of pseudostreamer structures that are associated with the Ulysses observations in these two Carrington rotations. Conclusions and discussion are presented in section 4.

\section{Extreme Solar Wind Proton Flux Observed in Two Solar Minima}

\subsection{Observations}

[6] The Ulysses mission observed the solar wind for more than 18 years, covering the two most recent solar minima. Figure 3 represents Ulysses' heliocentric distance and heliographic latitude (top panel) compared with sunspot number (bottom panel) in its life span. In order to have comparable periods of study for the two minima, we choose intervals that meet three criteria: (1) they have the same duration in time (as highlighted by yellow rectangles in Figure 3); (2) they cover the same latitudes in Ulysses' orbit (blue lines in the top panel of Figure 3); (3) they extend from the declining phases of the cycle to minima (see bottom panel of Figure 3 ).

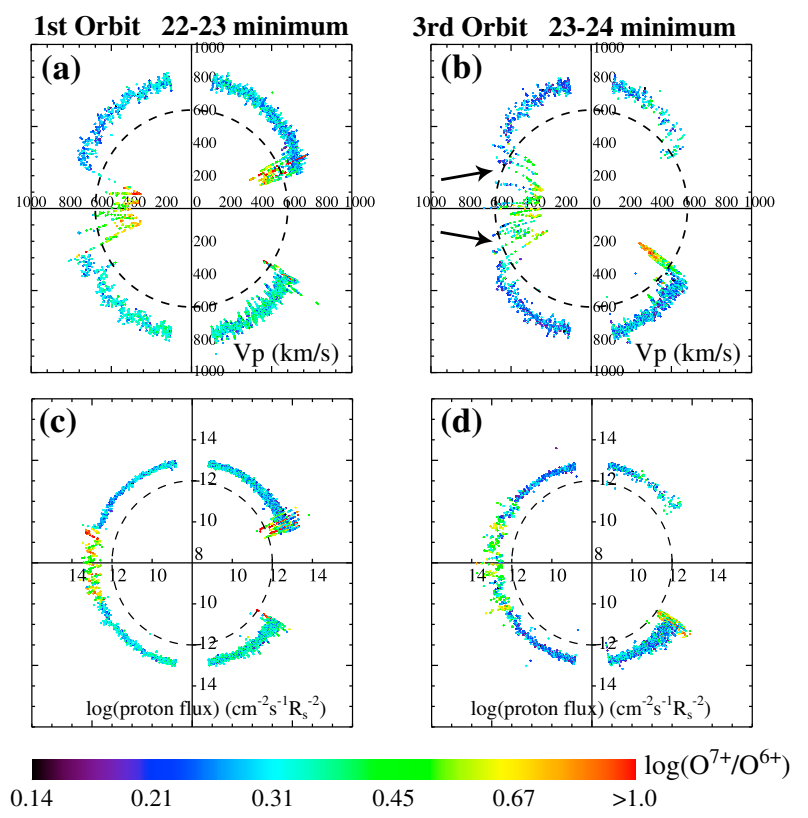

Figure 2. (a and b) Polar plots of solar wind proton speed and (c and d) proton flux color coded by $\mathrm{O}^{7+} / \mathrm{O}^{6+}$ ratio from Ulysses' first (in Figures $2 \mathrm{a}$ and $2 \mathrm{c}$ ) and third (in Figures $2 \mathrm{~b}$ and $2 \mathrm{~d}$ ) orbits. Time goes in clockwise direction, starting from the bottom-right quadrant. Black arrows point at the intermediate speed wind at the cycle 23-34 minimum. 


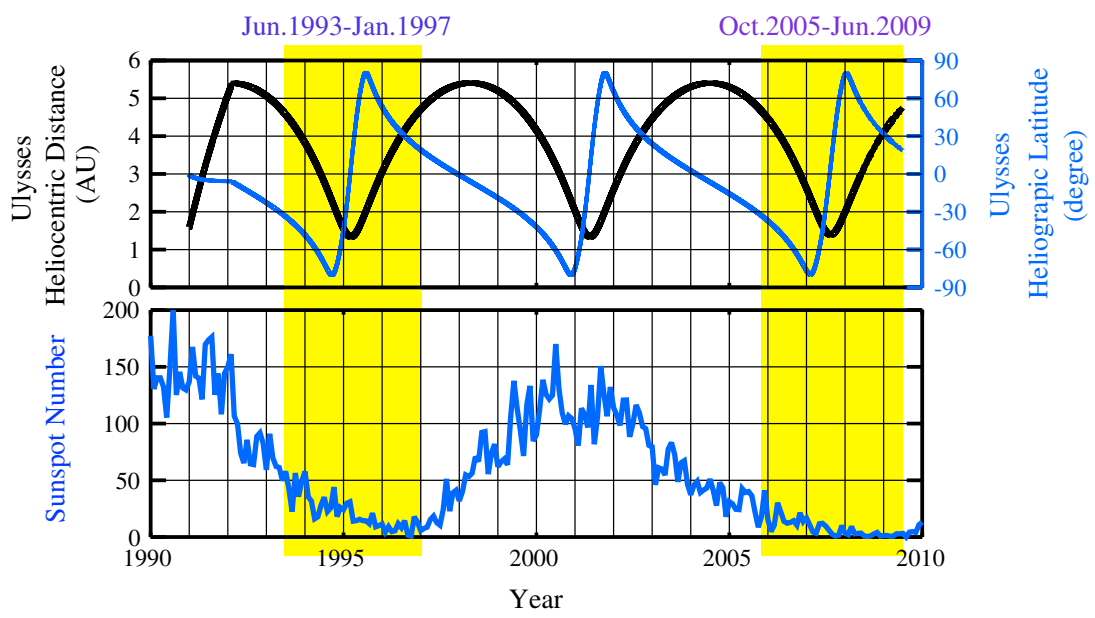

Figure 3. (top) Ulysses' heliocentric distance and heliographic latitude compared to (bottom) sunspot number. Intervals used in study are shown in yellow boxes: 1993.46-1997.03 (18 June 1993-10 January 1997) including cycle 22-23 minimum and 2005.83-2009.49 (30 October 2005-30 June 2009) including cycle 23-24 minimum.

[7] In this study, we analyze the solar wind proton speed and density measured by the SWOOPS (Solar Wind Observations over the Poles of the Sun) instrument onboard Ulysses [Bame et al., 1992]. In addition, we use the solar wind charge-state ratio, $\mathrm{O}^{7+} / \mathrm{O}^{6+}$, measured by the Solar Wind Ion Composition Spectrometer (SWICS) onboard Ulysses [Gloeckler et al., 1992] to indicate the electron temperature of the coronal source of the solar wind. Solar wind samples with different ionic charge-state composition are presumed to have different coronal origins. We derive the electron temperature based on the local thermal equilibrium model [Arnaud and Rothenflug, 1985; Mazzotta et al., 1998] and found the charge-state ratio monotonically increases with the electron temperature of the solar wind's coronal source. Also, note that we only concentrate on nontransient solar wind in this study; the interplanetary coronal mass ejections (ICMEs) are excluded by their ionic compositional signature introduced by Zhao et al. [2009].

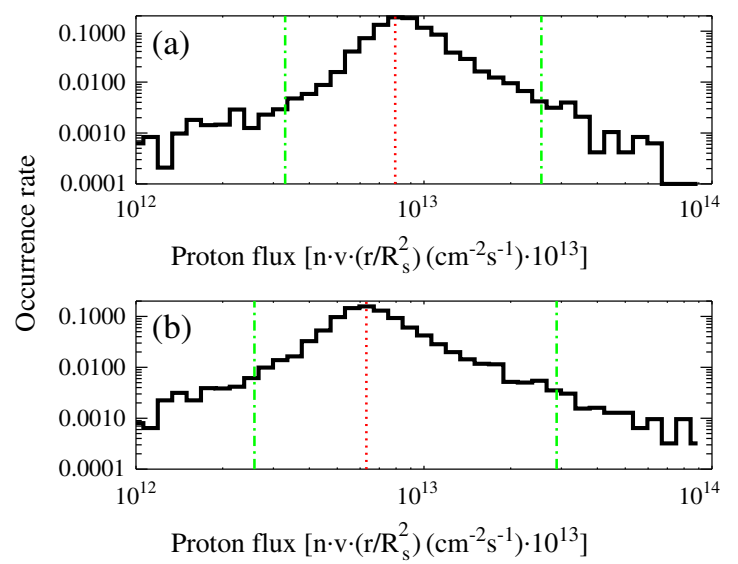

Figure 4. Distributions of proton flux in cycle (top) 22-23 minimum and (bottom) 23-24 minimum. Red dotted lines are the expectations of the distributions, and green dashdotted lines are the cutoffs that are three standard deviations away from the expectation.

\subsection{Proton Flux}

[8] Proton flux, as defined by the product of proton number density and proton speed, is expected to be constant along a flow tube and so can be determined for example at the coronal base [Fisk, 2003] or at the critical point [Parker, 1960]. In situ observation of Ulysses shows a global behavior of proton flux in its first (Figure 2c) and third (Figure 2d) orbits, including the periods when the Sun was in its cycle 22-23 and 23-24 activity minimum phases, respectively. In Figures 2c and $2 \mathrm{~d}$, the proton flux (normalized by Ulysses' heliocentric distance, $r^{2}$ ) of the solar wind in the two minima is polar plotted versus Ulysses' heliographic latitude and color coded by the $\mathrm{O}^{7+} / \mathrm{O}^{6+}$ ratio. The proton flux is essentially constant with heliographic latitude, especially for the cold (bluish) wind associated with open flux at higher latitudes. In middleto low-latitude regions, the variation of the solar wind proton flux is stronger. The dashed circles in Figures $2 \mathrm{c}$ and $2 \mathrm{~d}$ mark where the proton flux is $10^{12} \mathrm{~cm}^{-2} \mathrm{~s}^{-1} R_{s}^{-2}$. We find the extreme-proton-flux wind by defining its upper and lower limits. These limits represent three standard deviations from the peak expectation value of the proton flux distribution, where the standard deviations to the right and left of the peak are calculated independently to take into account the skew of the distributions (Figure 4). The values of these cutoffs are shown in Table 1. All the values having proton flux above (below) the upper (lower) limit can be considered as "extreme-high (low)" proton-flux wind. From Ulysses' point of view, most of these extreme-proton-flux winds are distributed around heliographic middle latitudes.

[9] The Ulysses proton flux polar plotted in Figures $2 \mathrm{c}$ and $2 \mathrm{~d}$ shows a distribution versus heliographic latitude. A more

Table 1. Cutoffs for Extreme Proton Flux in Two Solar Minima, Derived From Figure 4

\begin{tabular}{lcc}
\hline & 22-23 Minimum & 23-24 Minimum \\
\hline Proton flux $\left(\mathrm{cm}^{-2} \mathrm{~s}^{-1} R_{s}^{-2}\right)$ & $9.89 \pm 5.47 \times 10^{12}$ & $8.11 \pm 6.43 \times 10^{12}$ \\
Low cutoff & $3.3 \times 10^{12}$ & $2.6 \times 10^{12}$ \\
High cutoff & $2.6 \times 10^{13}$ & $2.9 \times 10^{13}$ \\
\hline
\end{tabular}




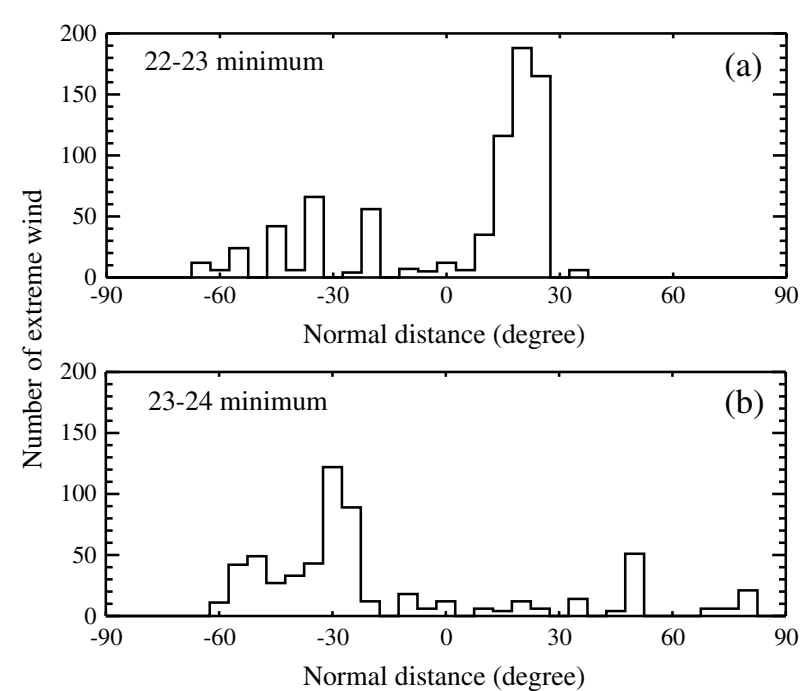

Figure 5. Distribution of extreme-proton-flux solar wind along the normal distance (Ds) relative to the HCS on 2.5 solar radii surface (source surface) in the cycle (a) 22-23 solar minimum and (b) 23-24 minimum.

specific quantity in understanding how the solar wind relates to the coronal and heliospheric magnetic field structure is the normal distance (Ds) from the solar wind source to the HCS. As described in Zhao et al. [2009], we utilize PFSS model to establish the location of the HCS and then map the in situ Ulysses observation to 2.5 solar radii (Rs), the traditional "source surface" upper boundary condition where coronal fields become radial. On the source surface, we calculate the normal distance along the great arc that connects the solar wind source position to the local HCS. This so-called "ballistic" map-back technique implicitly assumes that the solar wind plasma travels radially from the source surface to the spacecraft with a constant speed that is defined as the measured proton speed at Ulysses [Neugebauer et al., 2002; Zhao et al., 2009; Zhao and Fisk, 2011]. It has known shortcomings, especially near interfaces of fast and slow solar wind streams [Riley et al., 1999]. As discussed in Neugebauer et al. [2002], expected errors in source position are approximately $10^{\circ}$ longitude for Ulysses data, and since Ds is a normal distance, this represents an upper bound on error. Figure 5 shows the distribution of Ds calculated from the extreme-proton-flux solar wind source locations; HCS is located at Ds $=0$. The majority of the extreme-proton-flux wind sources come from middle to high latitudinal distances to HCS, with peaks at $\sim 20^{\circ}$ for $22-23$ minimum and $\sim 30^{\circ}$ in 23-24 minimum and with additional higher distance sources for the more recent minimum. These distributions indicate that the extreme-proton-flux wind originates in the intermediate zone lying between the classic bimodal HCS and polarcoronal-hole-associated wind. To examine this more closely, we now turn to the in situ observed properties of this subpopulation of the wind.

\subsection{In Situ Observed Properties of Extreme-Proton-Flux Solar Wind}

[10] The strong anticorrelation between electron temperature and proton speed [Gloeckler et al., 2003] has been argued to be evidence for interchange reconnection as an

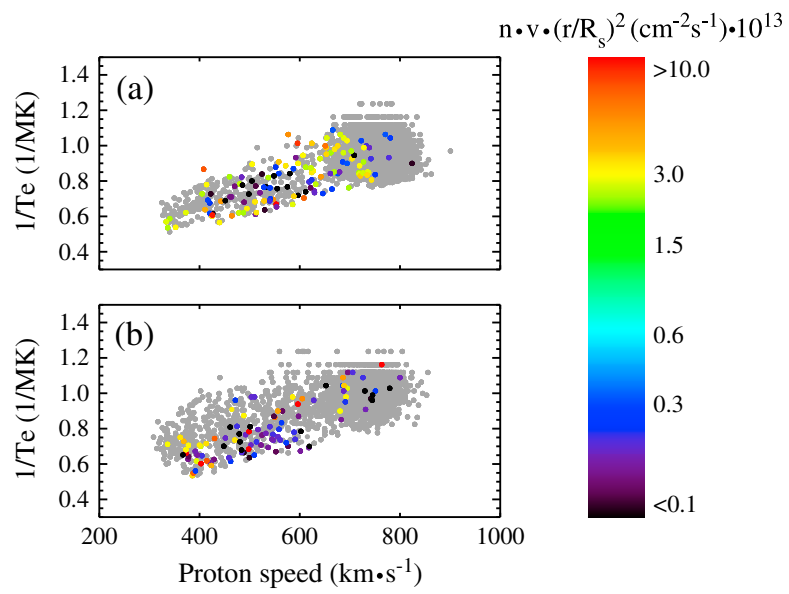

Figure 6. Comparison of electron temperature (derived from $\mathrm{O}^{7+} / \mathrm{O}^{6+}$ ratio) versus proton velocity in the (a) cycle 22-23 minimum and (b) cycle 23-24 minimum. Color coding is by proton flux, normalized by Ulysses radial distance. Population of extreme-low (blue-purple-black colors) and extreme-high (yellow-orange-red colors) proton flux are shown. Gray points are the rest of the solar wind.

acceleration mechanism applicable to all nontransient solar wind [Fisk, 2003]. To check if the extreme-proton-flux wind shows this anticorrelation, we compare electron temperature derived from Ulysses $\mathrm{O}^{7+} / \mathrm{O}^{6+}$ and proton speed for the two solar minima (Figure 6). We color code the extreme data by proton flux and plot the rest of the wind in gray. It is clear that the extreme-proton-flux wind follows the same anticorrelation trend as the rest of the solar wind in the two solar minima, which indicates those extreme winds have the same acceleration mechanism as the bulk of the solar wind.

[11] Figure 6 also shows that the extreme-proton-flux wind possesses a broad range of velocities and temperatures but
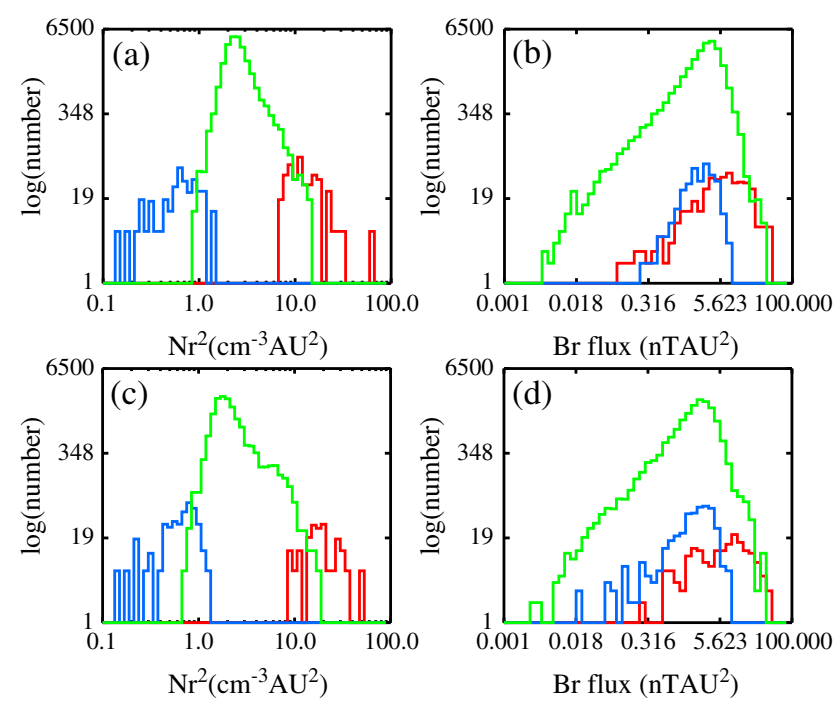

Figure 7. (a and c) Normalized proton density and (b and d) Br flux distribution of solar wind with extreme-high (red), extreme-low (blue), and intermediate (green) proton flux, in the cycle 22-23 minimum (in Figures $7 \mathrm{a}$ and $7 \mathrm{~b}$ ) and cycle 23-24 minimum (in Figures 7c and 7d). 
Table 2. Statistical Summary of the Comparison Between the Extreme-Proton-Flux Wind and Non-extreme Fast and Slow Wind in the 22-23 Minimum Observed by Ulysses

\begin{tabular}{ccccc}
\hline Mean \pm Standard Deviation & Extreme Low & Fast Wind & Slow Wind & Extreme High \\
\hline Proton speed $(\mathrm{km} / \mathrm{s})$ & $588 \pm 107$ & $774 \pm 25.6$ & $432 \pm 66.8$ & $582 \pm 117$ \\
Proton density $\left(\mathrm{cm}^{-3} r^{2}\right)$ & $0.713 \pm 0.319$ & $2.49 \pm 0.49$ & $6.15 \pm 2.7$ & $14.4 \pm 8.19$ \\
$B_{r}$ flux $\left(\mathrm{nT} \mathrm{AU}^{2}\right)$ & $0.086 \pm 0.075$ & $0.019 \pm 0.011$ & $0.156 \pm 0.11$ & $0.112 \pm 0.144$ \\
\hline
\end{tabular}

Table 3. Statistical Summary of the Comparison Between the Extreme-Proton-Flux Wind and Non-extreme Fast and Slow Wind in the 23-24 Minimum Observed by Ulysses

\begin{tabular}{ccccc}
\hline Mean \pm Standard Deviation & Extreme Low & Fast Wind & Slow Wind & Extreme High \\
\hline Proton speed $(\mathrm{km} / \mathrm{s})$ & $555 \pm 109$ & $756 \pm 24.2$ & $423 \pm 78.5$ & $495 \pm 124$ \\
Proton density $\left(\mathrm{cm}^{-3} r^{2}\right)$ & $0.679 \pm 0.306$ & $1.93 \pm 0.46$ & $4.90 \pm 3.63$ & $21.5 \pm 9.46$ \\
$B_{r}$ flux $\left(\mathrm{nT} \mathrm{AU}{ }^{2}\right)$ & $0.106 \pm 0.098$ & $0.011 \pm 0.008$ & $0.078 \pm 0.058$ & $0.138 \pm 0.153$ \\
\hline
\end{tabular}

is shifted to the left and for the most recent minimum downwards, indicating relatively slower proton speed and higher temperature than the rest of the solar wind. Extreme-high velocity and cold temperature (wind in the right-top corner) is not represented in this population.

[12] Besides the similarity of the extreme-proton-flux wind and the rest of the wind shown above, there are also distinctions between them, which are found in their measured proton density and radial open magnetic flux (Br) properties. Figure 7 shows the distribution of extreme-low (high) data in blue (red) and the rest of the wind in green. Proton density (normalized by Ulysses' heliocentric distance) is shown in Figures $7 \mathrm{a}$ and $7 \mathrm{c}$, and their average properties are presented in Tables 2 and 3 for the 22-23 minimum and 23-24 minimum, respectively, which shows that the extreme-high-proton-flux wind possesses very high density and extreme-lowproton-flux wind has low density relative to the rest of the wind. This indicates that the extremes in proton flux originate in the extremes in proton density.

[13] Figures $7 \mathrm{~b}$ and $7 \mathrm{~d}$ show the distribution of $\mathrm{Br}$ flux, and their average values are summarized in Tables 2 and 3 for the two minima. The extreme-high-proton-flux data (red) peak at a Br flux value greater than the rest of the solar wind (green). This is consistent with the theoretical argument that the amount of the open magnetic flux in a solar wind flux tube should be proportional to the solar wind mass flux along it [Fisk and Zhao, 2009; Zhao and Fisk, 2011]. Also, this is probably the consequence of the pile-up effect in the interface between fast and slow wind, i.e., corotating interaction regions, where fast wind pushes the slow wind in front of it, making both the plasma flux and B-field compressed. However, the extreme-low-proton-flux data (blue) peak at a $\mathrm{Br}$ flux value greater than or comparable to the peak in the rest of the wind, which does not seem to follow that trend; instead, the Br flux of this wind has a similar distribution with the entire population of $\mathrm{Br}$ at the two minima.

[14] Further, we compare the extreme-proton-flux wind with the nontransient and non-extreme slow wind and fast wind. In this study, we define slow wind as wind observed within heliographic latitude $10^{\circ}$ (Figure 2 ). In the two chosen intervals, Ulysses only observed slow wind in its fast latitudinal scans (left side of the polar plots in Figure 2). And we define fast wind as wind observed above a latitude of $60^{\circ}$. A statistical result of this comparison is summarized in Tables 2 and 3 for the two minima.

[15] We summarize the characteristics of the extremeproton-flux solar wind below:

[16] 1. It generally originates from sources middle-distant from the HCS (trending to higher distances for the most recent minimum).

[17] 2. It is associated with a broad range of velocities and electron temperatures but excludes very fast/cold wind.

[18] 3. It exhibits anticorrelation between electron temperature and proton velocity, as does the rest of the solar wind.

[19] 4. It has extreme proton density values relative to the rest of the solar wind.

[20] 5. The extreme-high-proton-flux wind has $\mathrm{Br}$ flux value greater than the rest of the solar wind; $\mathrm{Br}$ flux distribution of the extreme-low-proton-flux wind is very similar with the entire population of Br. Both extreme-high and extremelow winds do not possess the lowest values of $\mathrm{Br}$ flux.
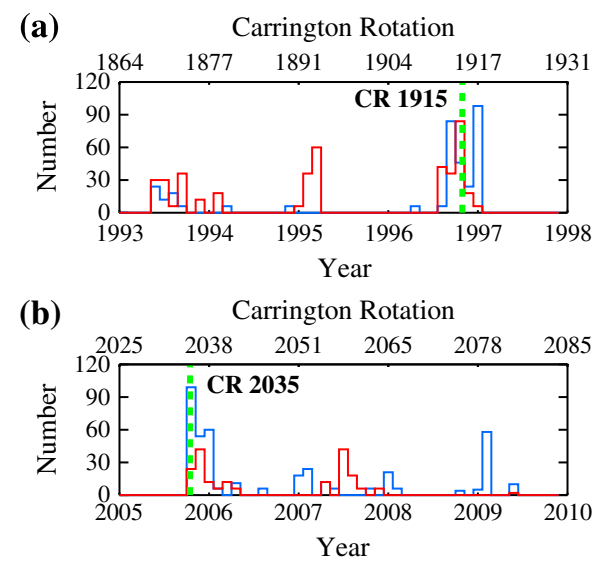

Figure 8. The temporal distributions of the extreme-high (low)-proton-flux wind are plotted along year (bottom $x$ axis) and Carrington number (top $x$ axis) in red (blue), in the (a) 22-23 minimum and (b) 23-24 minimum. Green vertical dotted lines mark the two interested rotations: CR 1915 and CR 2035. 

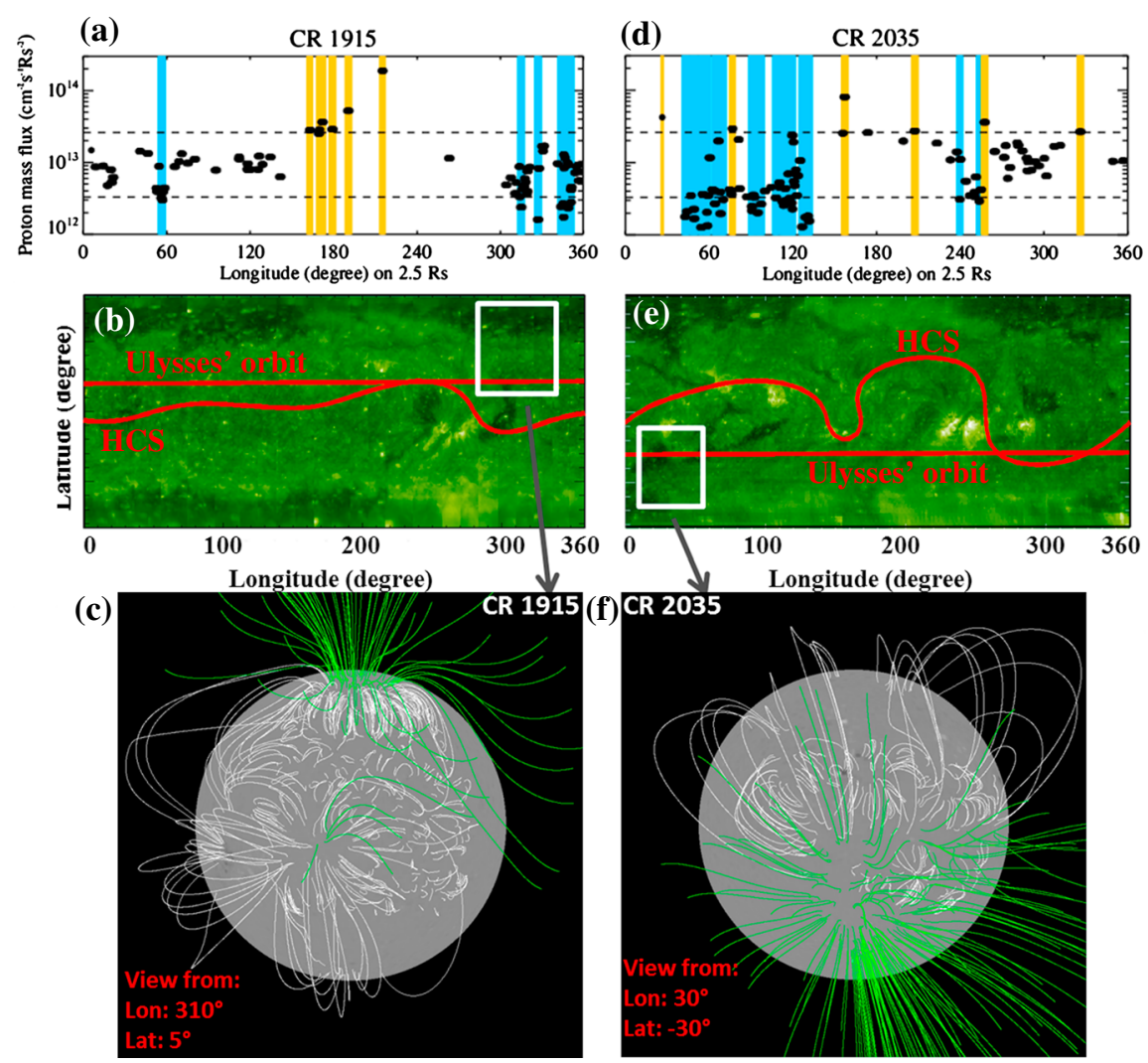

Figure 9. Case studies of (a, b, and c) CR 1915 and (d, e, and f) CR 2035. (top row) Solar wind proton flux observed by Ulysses and ballistically mapped on 2.5 Rs surface at CR 1915 and CR 2035. Horizontal dashed lines mark the low and high cutoffs as shown in Table 1. Blue (yellow) shades highlight the extreme-low (high) proton-flux wind. (middle row) SOHO EIT $195 \AA$ images in CR 1915 and CR2035, with Ulysses' orbit and HCS over plotted in red solid lines; white boxes highlighted the regions where Ulysses' observed extreme-high- and extremelow-proton-flux wind, and there were low-latitude coronal holes present. (bottom row) PFSS extrapolated field lines (closed in white and open in green) rooted in these two interested regions in CR 1915 and CR 2035.

\section{Case Study}

[21] The temporal distributions of the extreme-proton-flux wind are illustrated in Figures 8a and 8b. There is a temporal-spatial similarity in the locations of the extreme-high- and low-proton-flux points. This is consistent with the association between low-latitude coronal holes and pseudostreamers. For example, many of the 22-23 minimum extreme-proton-flux points come from CR 1913-1918 (fall of 1996) (Figure 8a) during which time low-latitude extensions of the north polar coronal hole were prevalent [Del Zanna and Bromage, 1999; Zhao et al., 1999; Posner et al., 1999]. Moreover, many of the 23-24 minimum extreme-proton-flux points, both low and high, came from Carrington rotations in 2005 and 2006 (e.g., CR 2035-2042) (Figure 8b), including time periods when both long-lived low-latitude coronal holes and pseudostreamers were present. We chose one Carrington rotation from each solar minimum for case studies: CR 1915 (Figures 9a, 9b, and 9c) in 22-23 minimum and CR 2035 (Figures 9d, 9e, and 9f) in 23-24 minimum, when there are extreme-proton-flux winds observed by Ulysses.

[22] The top row of Figure 9 shows the solar wind proton flux observed by Ulysses and ballistically mapped back on 2.5 Rs surface at CR 1915 (Figure 9a) and CR 2035 (Figure 9b). The middle row of Figure 9 represents SOHO EIT $195 \AA$ images for those two Carrington rotations, in which we over-plot HCS and Ulysses' orbits with red solid lines. Comparing the in situ extreme-proton-flux wind (Figure 9, top row) with the EIT images, we found in both rotations the times of recurring of extreme-proton-flux wind occur when there are long-lived low-latitude coronal holes between the HCS and polar coronal holes, e.g., longitude $300-360^{\circ}$ in CR1915 (Figure 9b) and longitude $20-120^{\circ}$ in CR 2035 (Figure 9e). We marked the regions of interest in the EIT images by white boxes, where Ulysses was passing between low-latitude coronal holes and polar holes. The bottom row of Figure 9 shows PFSS extrapolation (open field lines in green and close filed lines in white) in the two regions of interest. There are clearly closed fields (white lines) in between the polar hole and the low-latitude coronal hole that are not associated with the HCS, which can be pseudostreamers. Therefore, we believe it is very plausible that Ulysses was crossing the wind from both the long-lived low-latitude coronal holes and pseudostreamers and those winds possess extreme-proton-flux in these two rotations. 


\section{Conclusion and Discussion}

[23] Constant solar wind proton flux with heliolatitude implies a linear anticorrelation between proton density and velocity. In the simple, bimodal solar minimum picture, this is essentially what is expected: low-density, high-velocity wind coming from polar coronal holes and high-density, low-velocity wind associated with the HCS. Following this trend, we would expect the highest density in the solar wind to be associated most closely with the slowest velocity and the HCS and the lowest density in the solar wind to be associated with the highest velocity and the center of the polar hole. However, what we find instead is that these extremes in density are associated with sources that lie in between, in terms of both coronal source position and speed, and that they represent extremes in proton flux.

[24] The nature of the extreme-high-proton-flux wind agrees with the pseudostreamer-associated wind reported by Riley and Luhmann [2012] in terms of speed and density. The combination of the extreme-high and extreme-low-protonflux wind is also in agreement with the pseudostreamerassociated wind that Wang et al. [2012] reported; such wind could be a "hybrid" type of outflow which is in the middle between classical slow and fast solar wind, with intermediate proton speed but relatively high electron temperature.

[25] Whether extremes in mass flux originate at the solar source or in evolution in the solar wind remains to be seen. If they are associated with pseudostreamers, however, the extremes may have coronal origin. Using tomographic reconstructions of coronal streamers versus pseudostreamers is a promising avenue for establishing the properties at the coronal source [Nuevo et al., 2012; Vasquez, private communication]. On the other hand, since pseudostreamers are intrinsically linked to low-latitude coronal holes, we might expect fast/slow wind stream interactions to be a significant driver of rarefaction or compression. Indeed, the low mass flux population may be also associated with the so-called "solar wind disappearance" events [Janardhan et al., 2005].

[26] In conclusion, we find an interesting subset of the solar wind, possessing extremes in proton flux. These populations behave in some ways like the bulk of the solar wind but in other ways differently. These similarities and differences may help to distinguish between models of solar wind acceleration and to deepen our understanding of this fundamental physical process. The solar source of these extreme-proton-flux winds can be associated with the coronal pseudostreamer structures.

[27] Acknowledgments. This research was supported by the NASA Living With a Star Heliophysics Postdoctoral Fellowship Program, administered by the University Corporation for Atmospheric Research (UCAR). The National Center for Atmospheric Research (UCAR) is supported by the National Science Foundation. LAF was supported by NSF grant AGS-1043012.

[28] Philippa Browning thanks Rudolf von Steiger and another reviewer for their assistance in evaluating this paper.

\section{References}

Abramenko, V., V. Yurchyshyn, J. Linker, Z. Mikić, J. Luhmann, and C. O. Lee (2010), Low-latitude coronal holes at the minimum of the 23rd solar cycle, Astrophys. J., 712, 813-818, doi:10.1088/0004-637X/ $712 / 2 / 813$.

Arnaud, M., and R. Rothenflug (1985), An updated evaluation of recombination and ionization rates, Astron. Astrophys. Suppl. Ser., 60, 425-457.
Bame, S. J., D. J. McComas, B. L. Barraclough, J. L. Phillips, K. J. Sofaly, J. C. Chavez, B. E. Goldstein, and R. K. Sakurai (1992), The ULYSSES solar wind plasma experiment, Astron. Astrophys. Suppl. Ser., 92, 237-265.

Burgi, A., and J. Geiss (1986), Helium and minor ions in the corona and solar wind-Dynamics and charge states, Sol. Phys., 103, 347-383, doi:10.1007/BF00147835.

Crooker, N. U., S. K. Antiochos, X. Zhao, and M. Neugebauer (2012), Global network of slow solar wind, J. Geophys. Res., 117, A04104, doi:10.1029/2011JA017236.

Del Zanna, G., and B. J. I. Bromage (1999), The Elephant's Trunk: Spectroscopic diagnostics applied to SOHO/CDS observations of the August 1996 equatorial coronal hole, J. Geophys. Res., 104, 9753-9766, doi:10.1029/1998JA900067.

Dwivedi, B. N., A. Mohan, and K. Wilhelm (2000), On the electron temperatures, densities and hot ions in coronal hole plasma observed by Sumer on SOHO, Adv. Space Res., 25, 1751-1756, doi:10.1016/S0273-1177(99) 00586-4.

Fisk, L. A. (2003), Acceleration of the solar wind as a result of the reconnection of open magnetic flux with coronal loops, J. Geophys. Res., 108(A4), 1157, doi:10.1029/2002JA009284.

Fisk, L. A., and L. Zhao (2009), The heliospheric magnetic field and the solar wind during the solar cycle, in Universal Heliophysical processes IAU Symp., vol. 257, edited by N. Gopalswamy and D. F. Webb, pp. 109-120, Cambridge Univ. Press, Cambridge, U.K., doi:10.1017/ S1743921309029160.

Gibson, S. E., and L. Zhao (2012), A porcupine Sun? Implications for the solar wind and Earth, in IAU Symposium, vol. 286, edited by C. H. Mandrini and D. F. Webb, pp. 210-214, Cambridge Univ. Press, Cambridge, U.K., doi:10.1017/S1743921312004851.

Gloeckler, G., J. Geiss, H. Balsiger, P. Bedini, J. C. Cain, J. Fisher, L. A. Fisk, A. B. Galvin, F. Gliem, and D. C. Hamilton (1992), The Solar Wind Ion Composition Spectrometer, Astron. Astrophys. Suppl. Ser., 92, 267-289.

Gloeckler, G., T. H. Zurbuchen, and J. Geiss (2003), Implications of the observed anticorrelation between solar wind speed and coronal electron temperature, J. Geophys. Res., 108(A4), 1158, doi:10.1029/2002JA009286.

Gosling, J. T. (1997), Physical nature of the low-speed solar wind, in Robotic Exploration Close to the Sun: Scientific Basis, American Institute of Physics Conference Series, vol. 385, edited by S. R. Habbal, AIP Conf. Ser., 385. pp. 17-24, doi:10.1063/1.51743.

Hundhausen, A. J. (1972), Coronal expansion and solar wind in physics and chemistry, in Space, vol. 5, edited by A. J. Hundhausen, Springer, Berlin, Heidelberg.

Janardhan, P., K. Fujiki, M. Kojima, M. Tokumaru, and K. Hakamada (2005), Resolving the enigmatic solar wind disappearance event of 11 May 1999, J. Geophys. Res., 110, A08101, doi:10.1029/2004JA010535.

Kirk, M. S., W. D. Pesnell, C. A. Young, and S. A. Hess Webber (2009), Automated detection of EUV polar coronal holes during solar cycle 23, Sol. Phys., 257, 99-112, doi:10.1007/s11207-009-9369-y.

Landi, E., J. R. Gruesbeck, S. T. Lepri, and T. H. Zurbuchen (2012), New solar wind diagnostic using both in situ and spectroscopic measurements, Astrophys. J., 750, 159, doi:10.1088/0004-637X/750/2/159.

Mazzotta, P., G. Mazzitelli, S. Colafrancesco, and N. Vittorio (1998), Ionization balance for optically thin plasmas: Rate coefficients for all atoms and ions of the elements H to NI, Astron. Astrophys. Suppl. Ser., 133, 403-409, doi:10.1051/aas:1998330.

McComas, D. J., B. L. Barraclough, H. O. Funsten, J. T. Gosling, E. Santiago-Muñoz, R. M. Skoug, B. E. Goldstein, M. Neugebauer, P. Riley, and A. Balogh (2000), Solar wind observations over Ulysses' first full polar orbit, J. Geophys. Res., 105, 10,419-10,434, doi:10.1029/ 1999JA000383.

Neugebauer, M., P. C. Liewer, E. J. Smith, R. M. Skoug, and T. H. Zurbuchen (2002), Sources of the solar wind at solar activity maximum, J. Geophys. Res., 107(A12), 1488, doi:10.1029/2001JA000306.

Nuevo, F. A., Z. Huang, A. M. Vasquez, and R. A. Frazin (2012), A study of the thermodynamical properties of streamers and pseudostreamers, Abstract SH53A-2264 presented at 2012 Fall Meeting, AGU, San Francisco, Calif., 3-7 Dec.

Parker, E. N. (1960), The hydrodynamic treatment of the expanding solar corona, Astrophys. J., 132, 175, doi:10.1086/146910.

Posner, A., V. Bothmer, B. J. Thompson, H. Kunow, B. Heber, R. Müller-Mellin, A. J. Lazarus, A. Szabo, Z. Mikić, and J. A. Linker (1999), In-ecliptic CIR-associated energetic particle events and polar coronal hole structures: SOHO/COSTEP observations for the Whole Sun Month Campaign, J. Geophys. Res., 104, 9881-9890, doi:10.1029/98JA02654.

Riley, P., and J. G. Luhmann (2012), Interplanetary signatures of unipolar streamers and the origin of the slow solar wind, Solar Phys., 277, 355-373, doi:10.1007/s11207-011-9909-0.

Riley, P., et al. (1999), Relationship between Ulysses plasma observations and solar observations during the Whole Sun Month campaign, J. Geophys. Res., 104, 9871, doi:10.1029/1998JA900078. 


\section{ZHAO ET AL.: SOLAR WIND PROTON-FLUX EXTREMES}

Smith, E. J., and A. Balogh (1995), Ulysses observations of the radial magnetic field, Geophys. Res. Lett., 22, 3317-3320, doi:10.1029/95GL02826.

Smith, E. J., and A. Balogh (2008), Decrease in heliospheric magnetic flux in this solar minimum: Recent Ulysses magnetic field observations, Geophys. Res. Lett., 35, L22103, doi:10.1029/2008GL035345.

von Steiger, R., N. Schwadron, L. Fisk, J. Geiss, G. Gloeckler, S. Hefti, B. Wilken, R. Wimmer-Schweingruber, and T. Zurbuchen (2000), Composition of quasi-stationary solar wind flows from Ulysses/solar wind ion composition spectrometer, J. Geophys. Res., 105, 27,217-27,238.

von Steiger, R., T. H. Zurbuchen, J. Geiss, G. Gloeckler, L. A. Fisk, and N. A. Schwadron (2001), The 3-D heliosphere from the Ulysses and ACE Solar Wind Ion Composition Experiments, Space Sci. Rev., 97, 123-127, doi:10.1023/A:1011886414964.

de Toma, G. (2011), Evolution of coronal holes and implications for highspeed solar wind during the minimum between cycles 23 and 24, Sol. Phys., 274, 195-217, doi:10.1007/s11207-010-9677-2.

Wang, Y.-M., N. R. Jr.Sheeley, and N. B. Rich (2007), Coronal pseudostreamers, Astrophys. J., 658, 1340-1348, doi:10.1086/511416.

Wang, Y.-M., R. Grappin, E. Robbrecht, and N. R. Jr.Sheeley (2012), On the nature of the solar wind from coronal pseudostreamers, Astrophys. J., 749, 182, doi:10.1088/0004-637X/749/2/182.

Zhao, L., and L. Fisk (2011), Understanding the behavior of the heliospheric magnetic field and the solar wind during the unusual solar minimum between cycles 23 and 24, Sol. Phys., 274, 379-397, doi:10.1007/ s11207-011-9840-4.

Zhao, X. P., and D. F. Webb (2003), Source regions and storm effectiveness of frontside full halo coronal mass ejections, J. Geophys. Res., 108(A6), 1234, doi:10.1029/2002JA009606.

Zhao, X. P., J. T. Hoeksema, and P. H. Scherrer (1999), Changes of the boot-shaped coronal hole boundary during Whole Sun Month near sunspot minimum, J. Geophys. Res., 104, 9735-9752, doi:10.1029/ 1998JA900010.

Zhao, L., T. H. Zurbuchen, and L. A. Fisk (2009), Global distribution of the solar wind during solar cycle 23: ACE observations, Geophys. Res. Lett., 36, 14104, doi:10.1029/2009GL039181.

Zhao, L., S. E. Gibson, and L. A. Fisk (2012), Solar wind proton flux extremes and their association with pseudostreamers, in paper presented at Solar Wind 13: Thirteenth International Solar Wind Conference, Natl. Sci. Found., Kona-Kailua, Hawaii.

Zurbuchen, T. H., S. Hefti, L. A. Fisk, G. Gloeckler, and N. A. Schwadron (2000), Magnetic structure of the slow solar wind: Constraints from composition data, J. Geophys. Res., 105, 18,327-18,336, doi:10.1029/1999JA000427.

Zurbuchen, T. H., L. A. Fisk, G. Gloeckler, and R. von Steiger (2002), The solar wind composition throughout the solar cycle: A continuum of dynamic states, Geophys. Res. Lett., 29(9), 1352, doi:10.1029/ 2001 GL013946. 